\title{
Association of iron rim lesions with brain and cervical cord volume in relapsing multiple sclerosis
}

\author{
Claudia E. Weber ${ }^{1} \cdot$ Julia Krämer $^{2} \cdot$ Matthias Wittayer $^{1} \cdot$ Johannes Gregori $^{3} \cdot$ Sigurd Randoll $^{3} \cdot$ Florian Weiler $^{4}$. \\ Stefan Heldmann ${ }^{4}$. Christina Roßmanith ${ }^{1} \cdot$ Michael Platten $^{1} \cdot$ Achim Gass $^{1} \cdot$ Philipp Eisele $^{1}$
}

Received: 25 March 2021 / Revised: 22 June 2021 / Accepted: 22 July 2021 / Published online: 22 September 2021

(C) The Author(s) 2021, corrected publication 2022

\begin{abstract}
Objectives In multiple sclerosis (MS), iron rim lesions (IRLs) are indicators of chronic low-grade inflammation and ongoing tissue destruction. The aim of this study was to assess the relationship of IRLs with clinical measures and magnetic resonance imaging (MRI) markers, in particular brain and cervical cord volume.

Methods Clinical and MRI parameters from 102 relapsing MS patients (no relapses for at least 6 months, no contrastenhancing lesions) were included; follow-up data obtained after 12 months was available in 49 patients. IRLs were identified on susceptibility-weighted images (SWIs). In addition to standard brain and spinal cord MRI parameters, normalised cross-sectional area (nCSA) of the upper cervical cord was calculated.

Results Thirty-eight patients had at least one IRL on SWI MRI. At baseline, patients with IRLs had higher EDSS scores, higher lesion loads (brain and spinal cord), and lower cortical grey matter volumes and a lower nCSA. At follow-up, brain atrophy rates were higher in patients with IRLs. IRLs correlated spatially with T1-hypointense lesions.

Conclusions Relapsing MS patients with IRLs showed more aggressive MRI disease characteristics in both the cross-sectional and longitudinal analyses.

Key Points

- Multiple sclerosis patients with iron rim lesions had higher EDSS scores, higher brain and spinal cord lesion loads, lower cortical grey matter volumes, and a lower normalised cross-sectional area of the upper cervical spinal cord.

- Iron rim lesions are a new lesion descriptor obtained from susceptibility-weighted MRI. Our data suggests that further exploration of this lesion characteristic in regard to a poorer prognosis in multiple sclerosis patients is warranted.
\end{abstract}

Claudia E. Weber and Julia Krämer are first authors and contributed equally to this article.

Achim Gass and Philipp Eisele are senior authors and contributed equally to this article.

Achim Gass

achim.gass@medma.uni-heidelberg.de

1 Department of Neurology, Medical Faculty Mannheim and Mannheim Center for Translational Neurosciences (MCTN), University of Heidelberg, Theodor-Kutzer-Ufer 1

-3, 68167 Mannheim, Germany

2 Department of Neurology With Institute of Translational Neurology, University Hospital Münster,

Albert-Schweitzer-Campus 1; Gebäude A1, Westturm, Ebene

5, 48149 Münster, Germany

3 Mediri GmbH, Eppelheimer Straße 113, 69115 Heidelberg, Germany

4 Fraunhofer MEVIS, Am Fallturm 1, 28359 Bremen, Germany 
Keywords Multiple sclerosis $\cdot$ Magnetic resonance imaging $\cdot$ Spinal cord

$\begin{array}{ll}\text { Abbreviations } \\ \text { DGM } & \text { Deep grey matter } \\ \text { DMT } & \text { Disease-modifying therapy } \\ \text { EDSS } & \text { Expanded Disability Status Scale } \\ \text { FLAIR } & \text { Fluid-attenuated inversion recovery } \\ \text { FOV } & \text { Field of view } \\ \text { IRLs } & \text { Iron rim lesions } \\ \text { LV } & \text { Lesion volume } \\ \text { MPRAGE } & \text { Magnetization-prepared rapid acquisition } \\ & \text { gradient-echo } \\ \text { MRI } & \text { Magnetic resonance imaging } \\ \text { MS } & \text { Multiple sclerosis } \\ \text { NAGM } & \text { Normal-appearing grey matter } \\ \text { NAWM } & \text { Normal-appearing white matter } \\ \text { nCSA } & \text { Normalised cross-sectional area } \\ \text { PBVC } & \text { Percentage brain volume change } \\ \text { ST } & \text { Slice thickness } \\ \text { SWI } & \text { Susceptibility-weighted imaging } \\ \text { TE } & \text { Echo time } \\ \text { TI } & \text { Inversion time } \\ \text { TR } & \text { Repetition time }\end{array}$

\section{Introduction}

In multiple sclerosis (MS), magnetic resonance imaging (MRI) allows the requested demonstration of dissemination in time and space [1] and plays an important role in disease monitoring. Even though focal T2-hyperintense lesions represent a characteristic hallmark of MS pathology, T2 lesion load does not correlate with disability [2].

Recently, there has been an increasing interest in "chronic active" MS lesions that are associated with higher disease severity and ongoing tissue destruction as an imaging biomarker of disease progression [3-8]. Histopathologically, chronic active lesions are characterised by a self-sustained low degree of chronic inflammation, active myelin breakdown, neurodegeneration, axonal loss, and progressive tissue matrix damage driven by a rim of iron-laden activated microglia/macrophages and reactive astrocytes at the lesion edge $[4,9]$. Previous combined MRI/post-mortem studies demonstrated that these pathophysiological conditions can be visualised as hypointense rims on susceptibility-weighted imaging (SWI) [4, 6, 7], termed "iron rim lesions" (IRLs) [6, 10]. While previous studies reported an association between IRLs and brain volumes [6], the relationship between IRLs and spinal cord volumes has not been investigated until now.
As part of the central nervous system, the spinal cord represents an eloquent site of MS pathology and 80-90\% patients show focal lesions and tissue abnormalities on spinal cord MRI [11]. Previous MRI studies demonstrated the association of spinal cord atrophy and disability [12-16]. Spinal cord atrophy is routinely measured as the crosssectional area (CSA) at high cervical levels that provide reproducible results and are least affected by movements artefacts $[11,17]$.

The aims of this study were (i) to characterise differences of brain and spinal cord MRI readouts and (ii) to investigate the 1-year progression of brain and spinal cord atrophy in MS patients with IRLs compared to patients without those lesions. We hypothesise that IRLs correlate with clinical disability and MRI markers, in particular brain and cervical cord volume.

\section{Materials and methods}

\section{Patients}

\section{Cross-sectional investigation}

We retrospectively screened our database to identify patients diagnosed with relapsing MS according to the 2010 diagnostic criteria [1] fulfilling the following inclusion criteria (Fig. 1): (i) 18-65 years of age; (ii) a 3-T brain and spinal cord MRI dataset acquired in a single session including a 3D magnetization-prepared rapid acquisition gradient-echo (MPRAGE) sequence (brain) covering the upper portion of the cervical spinal cord (C1/2), a 3D fluid-attenuated inversion recovery (FLAIR) dataset (brain), SWI (brain), and sagittal T2-weighted cervical spinal cord images; (iii) no clinical relapse, no disability progression for at least 6 months; (iv) existing disease-modifying therapy (DMT) or without DMT for at least 6 months, (v) no use of corticosteroids 6 months prior; and (vi) no acute contrast-enhancing lesions (brain and spinal cord MRI). Exclusion criteria were presence of neurological conditions other than MS, cardiovascular or respiratory disease, history of brain/spinal cord trauma, severe cord compression, and missing or unsatisfactory MRI data. On the day of the MRI examinations, all patients underwent a comprehensive clinical assessment including the Expanded Disability Status Scale (EDSS) by trained neurologists. 


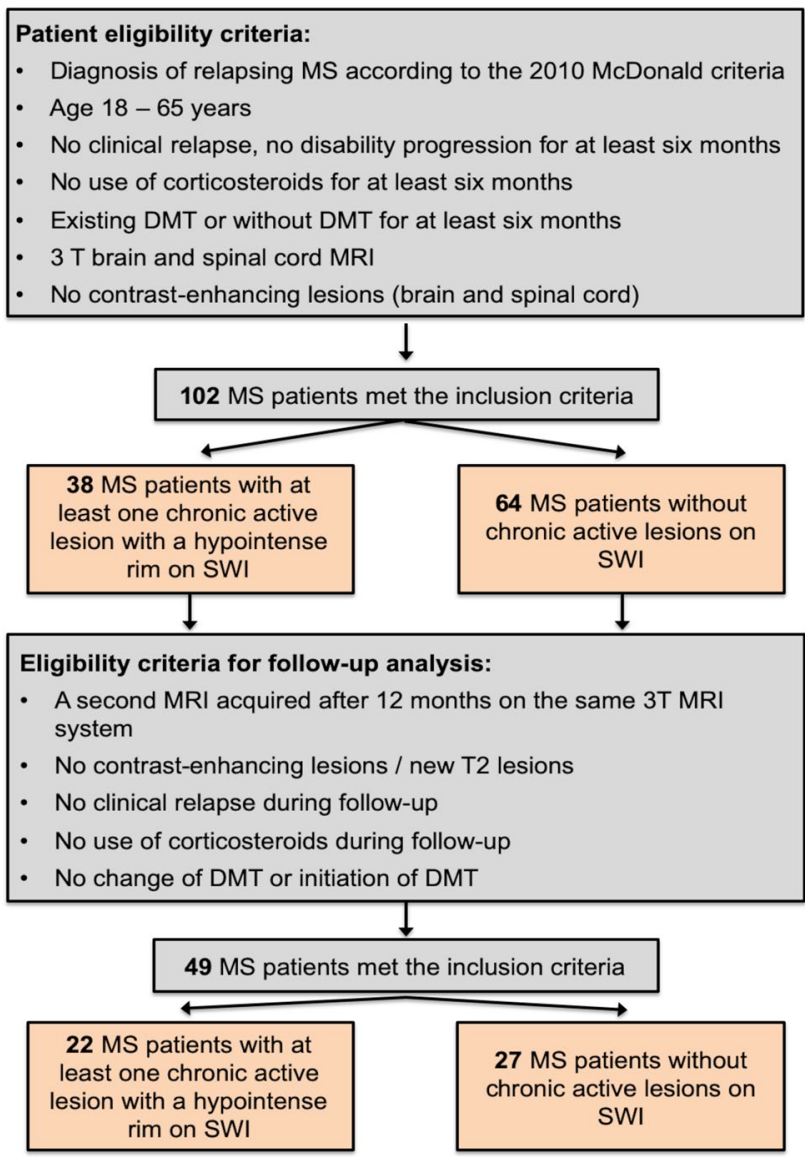

Fig. 1 Flowchart summarising how patients were selected for the study. Abbreviations: DMT, disease-modifying therapy; MS, multiple sclerosis; SWI, susceptibility-weighted imaging

\section{Longitudinal investigation}

For all patients included in the cross-sectional study, we screened if patients were eligible for a longitudinal follow-up substudy. To be included in the longitudinal study, patients had to fulfil the following inclusion criteria (Fig. 1): a second MRI acquired after 12 months follow-up on the same 3-T MRI system using identical imaging parameters, absence of acute contrastenhancing or new T2-hyperintense lesions, no clinical relapse, and no use of corticosteroids and no change of DMT (in case patients were on DMT at baseline) or initiation of DMT (in case patients were without DMT) during the follow-up period. Confirmed disability accumulation was defined as disability increase from study baseline, measured by EDSS (increase of $\geq 1.0$ points if baseline EDSS was $\leq 5.5$ or an increase $\geq 0.5$ for baseline EDSS > 5.5) [18]. Definition of converting to secondary progressive MS (SPMS) consisted a baseline EDSS $\geq 4.0$ and an increase $\geq 1.0$ points in patients with EDSS $\leq 5.5$ or an increase $\geq 0.5$ in patients with EDSS $\geq 6.0$ [19].

\section{Magnetic resonance imaging}

In all patients, MRI was performed on a 3-T MR system (MAGNETOM Skyra, Siemens Healthineers, 20-channel head coil) using a standardised protocol including a brain 3D MPRAGE sequence covering the upper portion of the cervical spinal cord (echo time $(\mathrm{TE})=2.49 \mathrm{~ms}$, repetition time $(\mathrm{TR})=1900 \mathrm{~ms}$, inversion time $(\mathrm{TI})=900 \mathrm{~ms}$, field of view $(\mathrm{FOV})=240 \mathrm{~mm}$, spatial resolution $=0.9 \times 0.9 \times 0.9$ $\left.\mathrm{mm}^{3}\right)$, a brain 3D FLAIR dataset $(\mathrm{TE}=398 \mathrm{~ms}$, $\mathrm{TR}=5000 \mathrm{~ms}, \mathrm{TI}=1800 \mathrm{~ms}, \mathrm{FOV}=240 \mathrm{~mm}$, resolution $\left.=0.5 \times 0.5 \times 0.9 \mathrm{~mm}^{3}\right)$, brain SWI $(\mathrm{TR}=27 \mathrm{~ms}$, $\mathrm{TE}=20 \mathrm{~ms}, \mathrm{FOV}=220 \mathrm{~mm}$, slice thickness $(\mathrm{ST})=1.5 \mathrm{~mm}$, voxel size $0.9 \times 0.9 \times 1.5 \mathrm{~mm}$ ) acquired after injection of gadoterate meglumine at a standard dose of $0.1 \mathrm{mmol} /$ $\mathrm{kg}, \mathrm{T} 1$-weighted images $(\mathrm{TR}=225 \mathrm{~ms}, \mathrm{TE}=2.5 \mathrm{~ms}$, $\mathrm{FOV}=220 \mathrm{~mm}, \mathrm{ST}=3 \mathrm{~mm}$, voxel size $0.7 \times 0.7 \times 3.0 \mathrm{~mm}$ ) acquired $10 \mathrm{~min}$ after contrast injection, and sagittal T2-weighted images $(\mathrm{TE}=108 \mathrm{~ms}, \mathrm{TR}=3.500 \mathrm{~ms}$, $\mathrm{FOV}=300 \mathrm{~mm}, \mathrm{ST}=3.0 \mathrm{~mm}$ ) with full coverage of the cervical cord.

\section{Spinal cord analysis}

Brain MPRAGE datasets were uploaded to the "mTRIAL 3.3" cloud service (mediri GmbH, www.mediri.com) that includes pseudonymization, automatic identification, and image quality assessment. Mean CSA at the $\mathrm{C} 1 / 2$ level in all patients was determined using the integrated component MSAAutoSegmentationCLI 1.1.7 (Fraunhofer MEVIS). The software uses a fully automated atlas-based approach for detection and segmentation of the upper cervical spinal cord from 3D brain MPRAGE images. In short, an atlas template is registered non-linearly to the subjects' image and spine segment definitions contained in the atlas template are transformed to the image. The pre-defined segments serve as a control for an iterative watershed-based segmentation, followed by fitting a bi-modal Gaussian mixture model with partial volume modelling to the histogram for volume measurement of the cord. The mean CSA is then obtained as the total cord volume divided by the cord length (Fig. 2) [20,21]. Baseline (and if available follow-up) CSA was normalised (nCSA) as suggested previously [12]. 


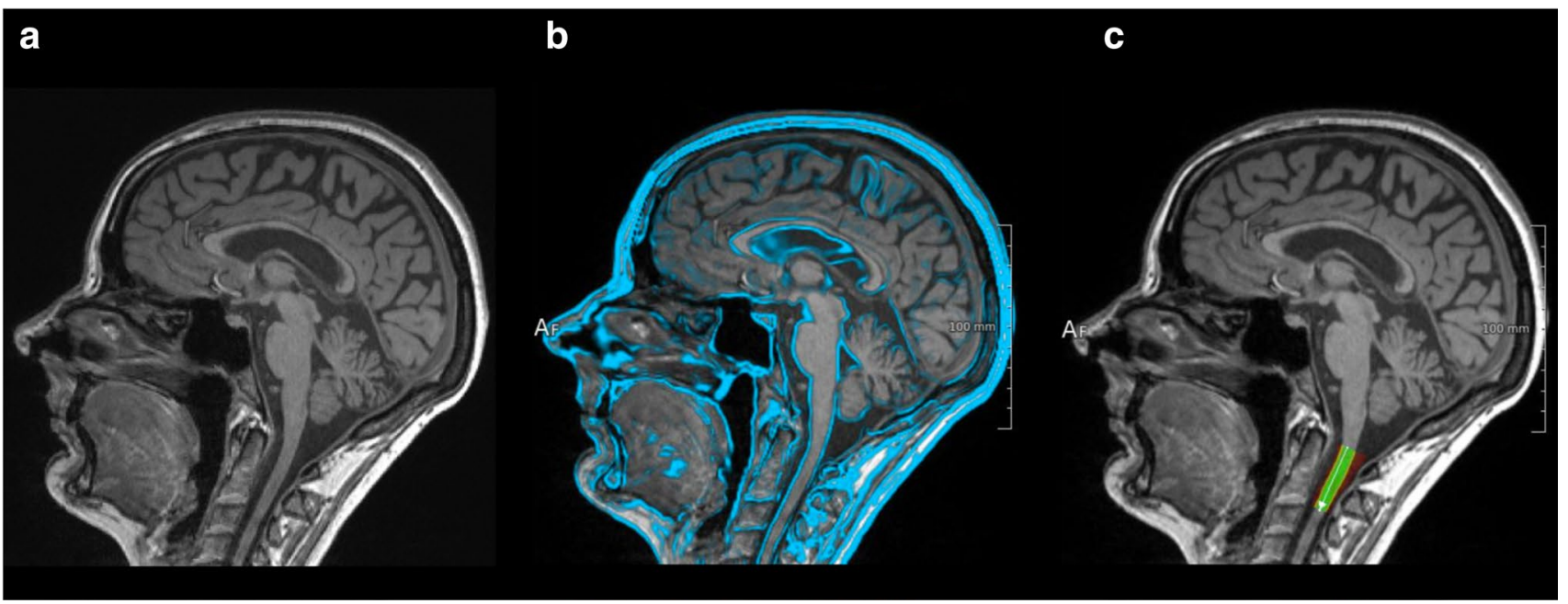

Fig. 2 Calculation of the cross-sectional area (CSA) from a 3D magnetization-prepared rapid acquisition gradient-echo (MPRAGE) sequence (a) covering the upper portion of the cervical cord. (b) An atlas template image is registered to the subjects' MPRAGE image. (c) Extraction of the mean area along the segmented spine

of high T1-weighted hypointense lesion volume, T1-weighted hypointense lesions were filled with the mean intensity value of the normal-appearing white matter present in the same slice of the lesion. Calculation of deep grey matter (DGM) volumes was performed with the subcortical brain segmentation tool FSL FIRST. Since the main focus of our study was the spinal cord, only the average of the total DGM volume (sum of bilateral putamen, caudate, globus pallidus, thalamus) and thalamic volume was included in our statistical analysis [13].

SWI images were evaluated for the presence of IRLs by consensus by two MRI MS trial and diagnostic readers with each $>15$ years of MR reading experience, unaware of the results of other MRI readouts, as described previously [28]: areas of hypointense ring-like signals at the lesion edge, encircling it fully or partially (see Fig. 3). Brain T2-hyperintense, T1-hypointense, and iron rim lesion volumes (LV) were quantified using the drawing tool of MRIcroGL. Heat maps representing relative probabilities of lesion subtypes (T2-hyperintense, T1-hypointense, IRLs) were computed over all patients. Therefore, baseline images were registered to the MNI152 template using the automated FSL FLIRT tool. The same registration was applied to the lesion masks. Computation of the heat maps was performed analogous to the spinal cord. 
Fig. 3 Representative examples of iron rim lesions (IRLs). Fluid-attenuated inversion recovery (FLAIR; left) and susceptibility-weighted images (SWI; right) demonstrate multiple IRLs (encircled and magnification)

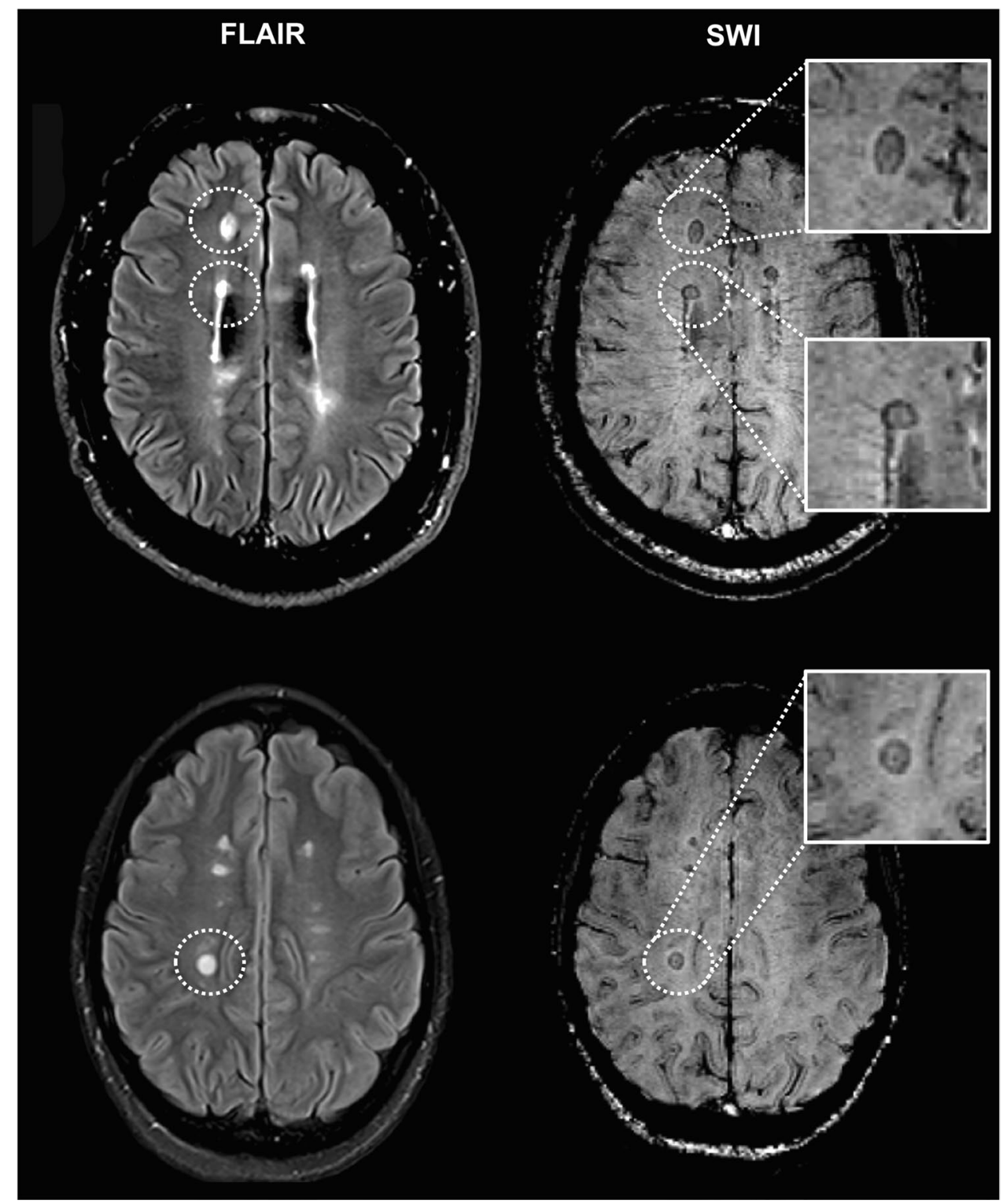

\section{Statistical analysis}

Statistical analysis was performed with $\mathrm{R}$ (version 4.0.2). Group comparisons (MS patients with versus without IRLs) of dichotomous variables across the study groups were analysed using chi-square tests, while unpaired variables were assessed using the Mann-Whitney $U$ test, since most of the variables were non-normally distributed. Level of significance was adjusted according to the Benjamini-Hochberg transformation despite the exploratory nature of the analysis.

For multivariate regression analysis of influence variables for the nCSA at the $\mathrm{C} 1 / \mathrm{C} 2$ level, the variable was z-transformed to achieve normal distribution. We performed a linear model regression to model the influence of clinical and imaging variables on the z-transformed nCSA using group (rim-positive vs. rim-negative), volume of IRLs, number of IRLs, DMT, high efficiency DMT (fingolimod, natalizumab), disease duration, cervical LV, T1- and T2-LV, age, gender, and EDSS at baseline as potential covariates. We applied an automated modelbuilding algorithm using MASS-package of R using Akaike information criterion (AIC) as goodness of fit indicator. The Kolmogorov-Smirnov test was used to confirm a normal distribution of the residuals.

Finally, to elucidate the clinical relevance of the findings, we conducted a correlation analysis for the variables EDSS 
Table 1 Mann-Whitney $U$ tests for unpaired variables for group comparisons

\begin{tabular}{|c|c|c|c|c|c|}
\hline & MS patients with IRLs & MS patients without IRLs & $W$ & $p$ value & Adjusted $p$ value \\
\hline Mean age, years (SD) & $\begin{array}{l}40.95 \\
(10.12)\end{array}$ & $\begin{array}{l}35.67 \\
(10.65)\end{array}$ & 867.5 & 0.01 & 0.38 \\
\hline Mean disease duration, years (SD) & $\begin{array}{l}8.58 \\
(8.85)\end{array}$ & $\begin{array}{l}3.48 \\
(3.29)\end{array}$ & 818.0 & 0.004 & 0.12 \\
\hline Median EDSS, baseline (range) & $\begin{array}{l}3 \\
(0-6.5)\end{array}$ & $\begin{array}{l}1 \\
(0-6)\end{array}$ & 523.5 & $<0.001$ & $<0.001$ \\
\hline Mean nCSA C1/2, $\mathrm{mm}^{2}(\mathrm{SD})$ & $\begin{array}{l}65.14 \\
(7.9)\end{array}$ & $\begin{array}{l}70.66 \\
(7.49)\end{array}$ & 1697.0 & $<0.001$ & 0.02 \\
\hline Mean volume, NAGM, mL (SD) & $739.74(56.81)$ & $790.89(53.57)$ & 1792.0 & $<0.001$ & 0.002 \\
\hline Mean volume, NAWM, mL (SD) & $722.43(61.58)$ & $745.16(49.54)$ & 1509.0 & 0.04 & 1 \\
\hline Mean volume, thalamus, $\mathrm{mL}$ (SD) & $\begin{array}{l}14.62 \\
(2.20)\end{array}$ & $\begin{array}{l}15.52 \\
(1.67)\end{array}$ & 1578.5 & 0.01 & 0.29 \\
\hline Mean volume, DGM, mL (SD) & $\begin{array}{l}33.45 \\
(4.52)\end{array}$ & $\begin{array}{l}35.32 \\
(3.55)\end{array}$ & 1542.0 & 0.02 & 0.58 \\
\hline Mean T2-LV, mL (SD) & $\begin{array}{l}9.39 \\
(11.99)\end{array}$ & $\begin{array}{l}2.87 \\
(5.34)\end{array}$ & 480.5 & $<0.001$ & $<0.001$ \\
\hline Mean T1-LV, mL (SD) & $\begin{array}{l}2.29 \\
(3.25)\end{array}$ & $\begin{array}{l}0.35 \\
(0.72)\end{array}$ & 482.5 & $<0.001$ & $<0.001$ \\
\hline Mean number of cervical lesions (SD) & $\begin{array}{l}2.34 \\
(1.66)\end{array}$ & $\begin{array}{l}0.98 \\
(1.29)\end{array}$ & 641.0 & $<0.001$ & $<0.001$ \\
\hline Mean cervical LV, mL (SD) & $\begin{array}{l}0.96 \\
(0.82)\end{array}$ & $\begin{array}{l}0.26 \\
(0.42)\end{array}$ & 510.0 & $<0.001$ & $<0.001$ \\
\hline
\end{tabular}

Abbreviations: DGM, deep grey matter; EDSS, Expanded Disability Status Scale; $I R L s$, iron rim lesions; $L V$, lesion volume; $N A G M$, normalappearing grey matter; $N A W M$, normal-appearing white matter; $n C S A$, normalised cross-sectional area; $S D$, standard deviation

at baseline and nCSA C1/C2 at baseline with other baseline variables. As most of the continuous parameters were nonnormally distributed, we used Spearman's rank correlations. As many tests were conducted, we adjusted the significance level according to the Benjamini-Hochberg transformation despite the exploratory nature of the analysis.

We then performed repeated measures ANOVAs analysing the influence of group and time point and the interaction of it on the variables reassessed for follow-up. Imaging measures that were reassessed in follow-up were referenced to the baseline in order to calculate the difference to the baseline.

\section{Standard protocol approvals, registrations, and patient consents}

The local ethics committee (2017-830R-MA) approved this study. Patient consent was waived due to the retrospective nature and lack of patient interaction.

\section{Results}

\section{Cross-sectional investigation}

Overall, 102 patients met the inclusion criteria. After lesion identification and classification, thirty-eight (37\%) patients had at least one IRL on SWI (mean 1.8 lesions per patient; range 1-7), whereas in the remaining sixty-four patients, no IRLs were found on SWI. All IRLs showed matching hypointensity on T1-weighted MRI.

The groups did not differ in terms of gender (patients with IRLs: 74\% female versus patients without IRLs: 75\% female; $p=0.93)$, DMT (79\% versus $80 \% ; p=1.0)$, high efficiency DMT (natalizumab, fingolimod; $37 \%$ versus $24 \%$; $p=0.34$ ), age $(40.95 \pm 10.12$ versus $35.67 \pm 10.65$ years; $p=0.38)$, and disease duration $(8.58 \pm 8.85$ versus $3.48 \pm 3.29$ years; $p=0.12$ ), whereas patients with IRLs had a significant higher median EDSS score (3.0 versus $1.0 ; p<0.001$; Table 1).

On baseline MRI, patients with IRLs had a significant lower mean nCSA C1/2, lower NAGM volumes, 
a higher T2- and T1-LV, a higher number of cervical lesions, and a higher cervical LV (Table 1). Figure 4 demonstrates a heat map of relative probabilities of spinal cord lesions. Relative probabilities of T2-hyperintense, T1-hypointense, and IRLs for all study participants are shown in Fig. 5.

\section{Linear model for the z-transformed CSA C1/C2}

The automated model building using MASS package in $\mathrm{R}$ with AIC as goodness of fit criterion resulted in a linear model including the variables group, gender, disease duration, cervical LV, and DMT as independent variables and the $\mathrm{z}$-transformed nCSA C1/C2 at baseline as the dependent variable. All other variables were removed from the model, as they did not improve the amount of explanation of the variance. The linear model was highly significant $(p<0.001)$ and showed a moderate explanation of variance $\left(R^{2}=0.2\right.$, adjusted $R^{2}=0.16$ ). Independent variables of the model are shown in Table 2. The model showed no multicollinearity. Residuals were not correlated to the independent variables and normally distributed by visual inspection of the histogram though the Shapiro-Wilk test showed tendencies towards skewness. Results of the correlation analysis between baseline EDSS and nCSA C1/C2 with the other baseline variables are shown in Table 3 .

\section{Longitudinal investigation}

Forty-nine patients (48\%) were included in the followup analysis. These included twenty-two patients with and twenty-seven patients without IRLs. Confirmed disability accumulation during follow-up was observed in seven patients $(14 \% ; 5 / 22$ patients with IRLs; $p=0.3$, repeated measures ANOVA for pre-post comparisons), and only one patient with IRLs fulfilled the definition of converting to SPMS [19]. During follow-up, PBVC was significantly higher in patients with IRLs $(-0.8 \pm 0.6 \%)$ compared to patients without IRLs $(-0.34 \pm 0.47 \% ; p=0.01)$. Differences of the cervical cord atrophy at the $\mathrm{C} 1 / 2$ level in patients with IRLs $(-2.44 \pm 0.95 \%)$ and patients without IRLs $(-1.73 \pm 0.96 \%)$ were not statistically significant ( $p=0.6$, repeated measures ANOVA for pre-post comparisons).

\section{Discussion}

In this study, we investigated the association between IRLs with clinical measures and MRI readouts, in particular brain and cervical spinal cord volumes. There are several points noteworthy in this regard.
There has been an increasing interest in IRLs as a new imaging biomarker of disease progression indicating ongoing disease activity in the absence of contrast enhancement [3-6]. Even though the in-plane voxel dimensions of the axial SWI used in our study were larger compared to previous studies [6,7], we found IRLs in $~ 40 \%$ of MS patients, a finding that is comparable to autopsy [29, 30] and MRI studies [28, 31], making SWI an interesting candidate for the detection of IRLs in a clinical setting. While post-mortem studies demonstrated that IRLs can be mainly found in progressive forms of MS [29, 32], our data and recent in vivo MRI studies show that a considerable amount of IRLs are also present in relapsing MS patients [3, 6]. Furthermore, our results are in line with previous studies that IRLs can mainly be found in patients with higher disease severity $[6,29]$. In our study, IRL volumes and the EDSS correlated moderately supporting the view that IRLs could serve as a marker of disease severity [3-6]. Interestingly and in line with other studies [3, 5, 33], IRLs were present in patients despite DMT including fingolimod or natalizumab supporting the notion that IRLs evolve as a consequence of macrophage/microglia-mediated inflammation that may be independent of newly developing lesions $[5,6]$.

All IRLs showed matching hypointensity on T1-weighted MRI and appear to be a subgroup of hypointense T1 lesions, which have been previously described as markers of pronounced tissue matrix damage [34]. Several studies have shown the correlation between iron rims and slowly expanding lesions on T1-weighted MRI [6, 10]. In our cross-sectional analysis, patients with IRLs had higher EDSS scores, higher brain T2- and T1-LV, and lower volumes of the NAGM, a finding that is in line with recent studies [6]. Furthermore, cervical nCSA, a novel spinal cord volume marker, was also smaller in patients with IRLs who also showed higher cervical cord T2 LV. Based on the multivariate modelling approach, this was not merely an effect of the longer disease duration in patients with and without IRLs. Iron rim LV and number of IRLs did not show any contribution to the explanation of variance at all and were removed from the model. Therefore, we hypothesise that the sheer presence of IRLs and the underlying pathophysiology might have an effect on spinal cord atrophy independent of the number and volume of IRLs. Of note, we found a significant, yet moderate, correlation of the nCSA and EDSS scores that is comparable to previous studies $[13,16]$. Interestingly, we also observed a significant correlation between cervical cord LV and disability, emphasising the relevance of demyelination 

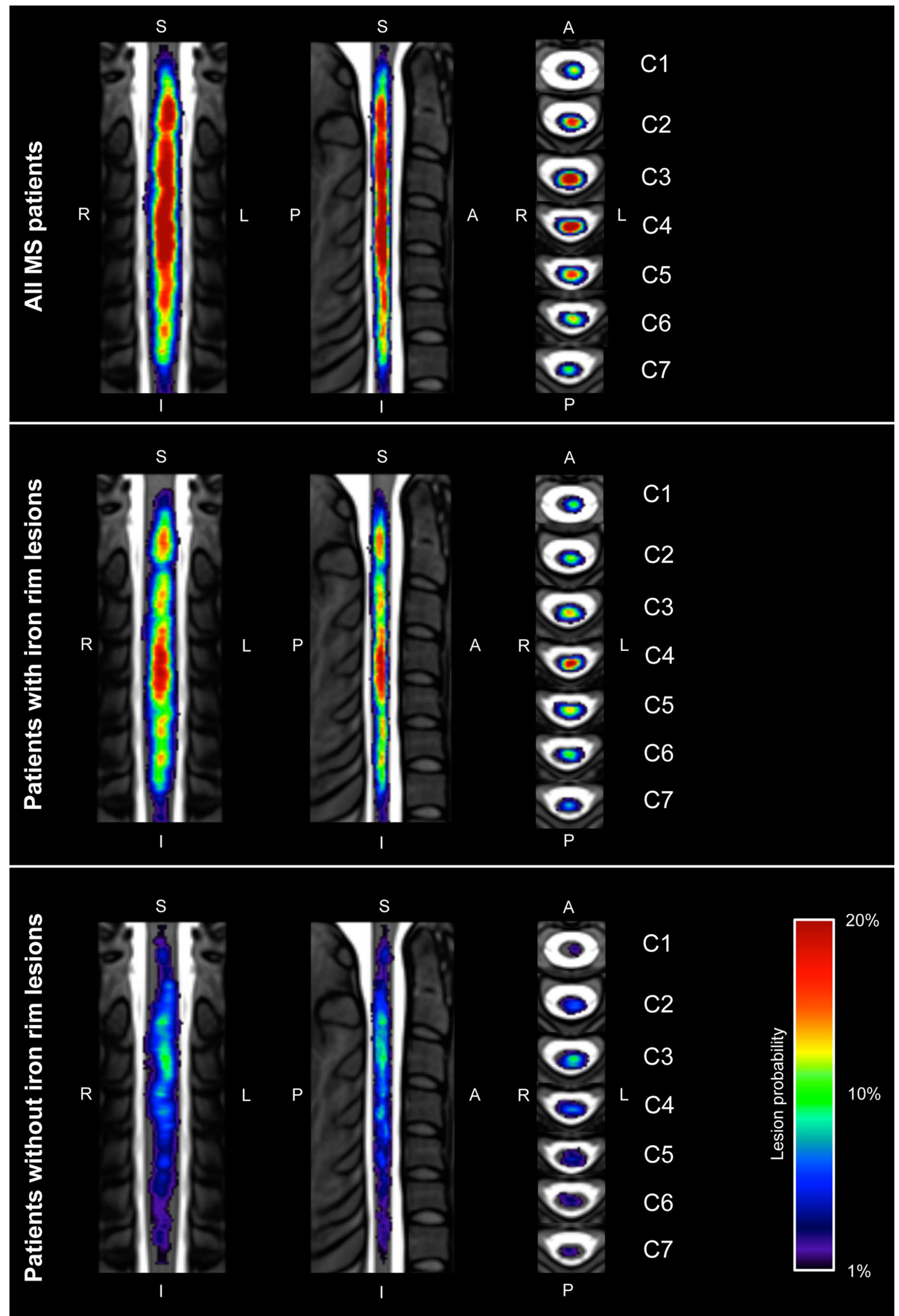
4Fig. 4 Heat map representation of relative probabilities of spinal cord lesions computed over all patients (top row), patients with iron rim lesions (middle row), and patients without iron rim lesions (bottom row) superimposed on the PAM50 spinal cord template. A, anterior; I, inferior; L, left; P, posterior; R, right; S, superior

in explaining disability [35] and underlining the heterogeneity and complexity of the disease. However, in our study, we used a sagittal T2-weighted sequence with a single long echo time for the detection of spinal cord lesion. Short tau inversion recovery sequences have a higher sensitivity [11]; therefore, the number of spinal cord lesions may be underestimated in our study.

In the 1-year longitudinal follow-up of 49 patients, the brain atrophy rate was higher in the IRL group. In line with previous observations, spinal cord atrophy rates were two to three times higher than brain atrophy [17]. Even though we found a trend of higher spinal cord atrophy rates in patients with IRLs, statistical significance was not reached, which is probably a result due to the relative short
Table 2 Linear model for the z-transformed normalised cross-sectional area at the $\mathrm{C} 1 / \mathrm{C} 2$ level. $* * p<0.01 ; * p<0.5$

\begin{tabular}{lrrlll}
\hline & Estimate & $t$ value & $p$ & \\
\hline Intercept & 0.29 & 0.99 & 0.33 & \\
Group & -0.64 & 0.23 & 0.005 & $* *$ \\
Gender & 0.42 & 1.95 & 0.05 & \\
Disease duration & -0.04 & -2.47 & 0.02 & $*$ \\
Cervical lesion volume & 0.24 & 1.56 & 0.12 & \\
Disease-modifying therapy & -0.35 & -1.5 & 0.14 & \\
\hline
\end{tabular}

follow-up period. This is certainly an important limitation of our study. Another potential limitation is that SWIs were acquired after contrast injection. Therefore, interpretation of our results should be done cautiously.

The presence of IRLs appears to indicate MRI and clinical features that point to more aggressive MS characteristics. Both conventional lesion measures and indicators of
Fig. 5 Heat map representation of relative probabilities of T2-hyperintense, T1-hypointense, and iron rim lesions computed over all patients included in the study superimposed on a MNI152 template

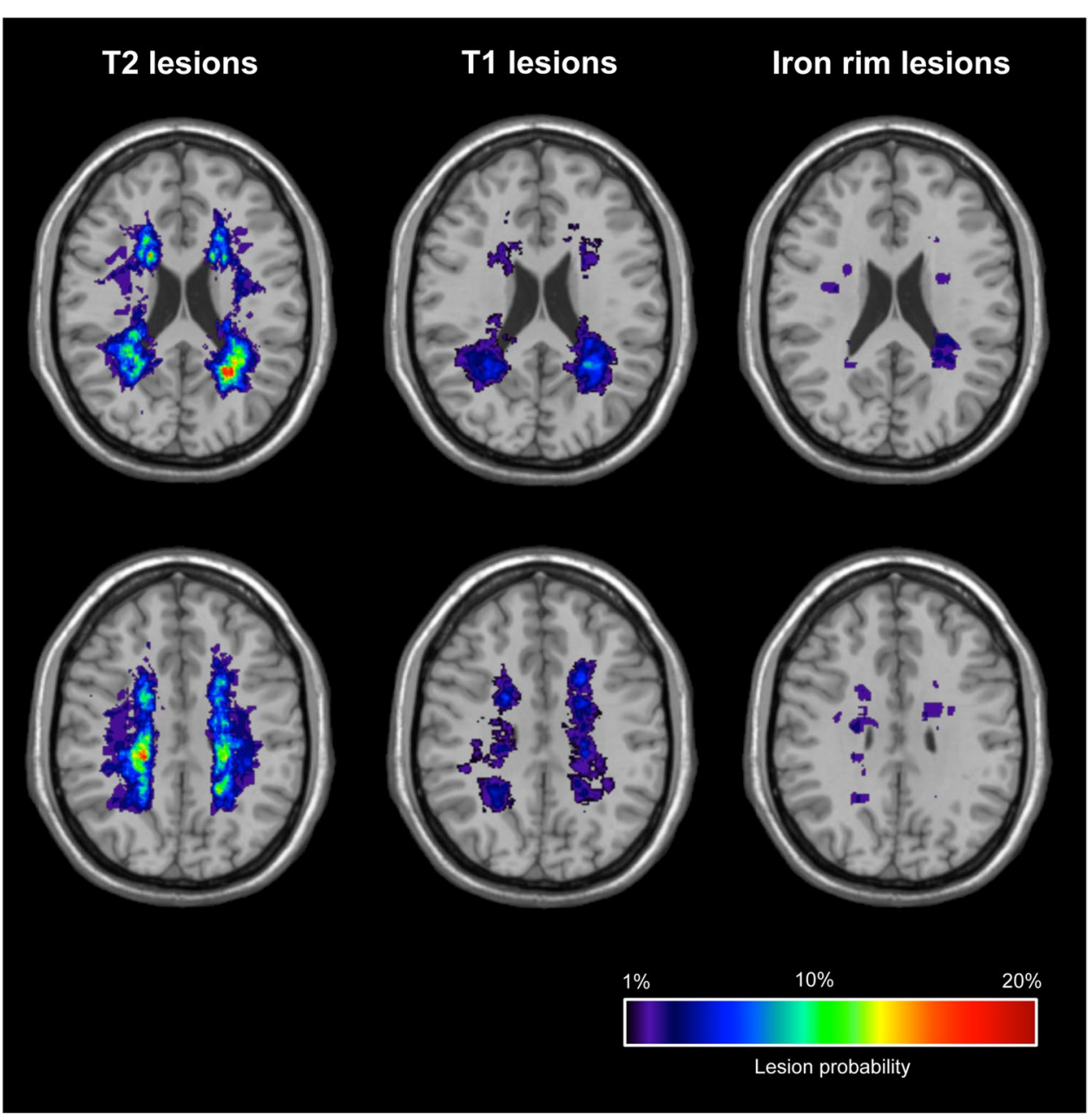


Table 3 Multivariate correlation analysis; $p$ values were adjusted using the Benjamini-Hochberg correction. Abbreviations: adj., adjusted; DGM, deep grey matter; EDSS, Expanded Disability Status Scale; IRLs, iron rim lesions; $L V$, lesion volume; $n C S A$, normalised cross-sectional area; $N A G M$, normal-appearing grey matter; $N A W M$, normal-appearing white matter; n.s. not significant

\begin{tabular}{lllllc}
\hline & \multicolumn{2}{l}{ EDSS baseline } & & \multicolumn{2}{l}{ nCSA C1/2 } \\
\cline { 2 - 3 } \cline { 6 - 7 } & $r$ & adj. $p$ & & $r$ & adj. $p$ \\
\hline Age & 0.36 & $<0.001$ & & n.s & \\
Disease duration & 0.43 & $<0.001$ & & -0.24 & 0.002 \\
EDSS baseline & - & & & -0.31 & 0.003 \\
nCSA C1/2 & -0.31 & 0.003 & & - & \\
Volume NAGM & -0.26 & 0.01 & & 0.2 & 0.05 \\
Volume NAWM & 0.39 & $<0.001$ & 0.34 & $<0.001$ \\
Volume thalamus & -0.26 & 0.01 & & 0.41 & $<0.001$ \\
Volume DGM & $\mathrm{n} . \mathrm{s}$ & & & 0.36 & $<0.001$ \\
T2 LV & 0.58 & $<0.001$ & -0.26 & 0.01 \\
T1 LV & 0.5 & $<0.001$ & & n.s & \\
IRL volumes & 0.48 & $<0.001$ & -0.31 & 0.002 \\
Cervical LV & 0.56 & $<0.001$ & & -0.21 & 0.04 \\
\hline
\end{tabular}

irreversible tissue change (brain and cord atrophy) were more pronounced in patients with IRLs. SWI has been advocated to be used to increase diagnostic specificity in MS and new study data may emphasise this [36]. The results of this study suggest that SWI may have prognostic value. SWI sequences are available at 3-T and, with a lower sensitivity, at 1.5-T systems and therefore could be used in clinical imaging. Previous longitudinal studies reported a slow expansion of IRLs $[4,6,10]$ and that iron rims persist and evolve over several years $[4,10]$. Therefore, larger imaging intervals (e.g. 2-3 years) for monitoring IRLs may be sufficient for clinical purposes.

Future longitudinal studies also including primary and secondary progressive MS patients and longer observation periods would be highly interesting.

In conclusion, IRLs are associated with more aggressive MRI disease characteristics in relapsing MS.

Funding Open Access funding enabled and organized by Projekt DEAL. The authors state that this work has not received any funding.

\section{Declarations}

Guarantor The scientific guarantor of this publication is Prof. Dr. Achim Gass, Department of Neurology, Medical Faculty Mannheim and Mannheim Center for Translational Neurosciences, University of Heidelberg, Theodor-Kutzer-Ufer 1 - 3, 68167 Mannheim, Germany.

Conflict of interest The authors of this manuscript declare relationships with the following companies: Dr. Krämer has received honoraria for lecturing from Biogen, Novartis, Genzyme, Merck, Mylan, and Teva and financial research support from Sanofi Genzyme and Novartis. Dr. Gregori is managing director of mediri GmbH. Prof. Platten has a consultant relationship with Novartis, Merck, and Genentech/Roche; has received non-personal, institutional honoraria from Medac, Merck, Novartis, TEVA, and Genentech/Roche; and has research agreements with Bayer Health Care. Prof. Gass has received honoraria for lecturing, travel expenses for attending meetings, and financial support for research from Bayer Schering, Biogen Idec, Merck Serono, Novartis, and TEVA Neurosciences. Dr. Eisele has received travel expenses from Bayer Health Care. The remaining authors declare no conflict of interest.

Statistics and biometry One of the authors (Dr. Matthias Wittayer) has significant statistical expertise.

Informed consent Written informed consent was waived by the institutional review board due to the retrospective nature and lack of patient interaction.

Ethical approval Institutional review board approval was obtained (2017-830R-MA).
Methodology
- retrospective
- iagnostic study
- performed at one institution

Open Access This article is licensed under a Creative Commons Attribution 4.0 International License, which permits use, sharing, adaptation, distribution and reproduction in any medium or format, as long as you give appropriate credit to the original author(s) and the source, provide a link to the Creative Commons licence, and indicate if changes were made. The images or other third party material in this article are included in the article's Creative Commons licence, unless indicated otherwise in a credit line to the material. If material is not included in the article's Creative Commons licence and your intended use is not permitted by statutory regulation or exceeds the permitted use, you will need to obtain permission directly from the copyright holder. To view a copy of this licence, visit http://creativecommons.org/licenses/by/4.0/.

\section{References}

1. Polman CH, Reingold SC, Banwell B et al (2011) Diagnostic criteria for multiple sclerosis: 2010 revisions to the McDonald criteria. Ann Neurol 69:292-302

2. Barkhof F (2002) The clinico-radiological paradox in multiple sclerosis revisited. Curr Opin Neurol 15:239-245

3. Elliott C, Wolinsky JS, Hauser SL et al (2019) Slowly expanding/evolving lesions as a magnetic resonance imaging marker of chronic active multiple sclerosis lesions. Mult Scler 25:1915-1925

4. Dal-Bianco A, Grabner G, Kronnerwetter C et al (2017) Slow expansion of multiple sclerosis iron rim lesions: pathology and 7 T magnetic resonance imaging. Acta Neuropathol 133:25-42

5. Elliott C, Belachew S, Wolinsky JS et al (2019) Chronic white matter lesion activity predicts clinical progression in primary progressive multiple sclerosis. Brain 142:2787-2799

6. Absinta M, Sati P, Masuzzo F et al (2019) Association of chronic active multiple sclerosis lesions with disability in vivo. JAMA Neurol 76:1474-1483

7. Absinta M, Sati P, Schindler M et al (2016) Persistent 7-Tesla phase rim predicts poor outcome in new multiple sclerosis patient lesions. J Clin Invest 126:2597-2609 
8. Weber CE, Kraemer M, Dabringhaus A et al (2021) Venous diameter changes in chronic active multiple sclerosis lesions. J Neuroimaging 31:394-400

9. Bagnato F, Hametner S, Yao B et al (2011) Tracking iron in multiple sclerosis: a combined imaging and histopathological study at 7 Tesla. Brain 134:3602-3615

10. Dal-Bianco A, Grabner G, Kronnerwetter C et al (2021) Longterm evolution of multiple sclerosis iron rim lesions in 7 T MRI. Brain 144:833-847

11. Gass A, Rocca MA, Agosta F et al (2015) MRI monitoring of pathological changes in the spinal cord in patients with multiple sclerosis. Lancet Neurol 14:443-454

12. Lin X, Tench CR, Turner B, Blumhardt LD, Constantinescu CS (2003) Spinal cord atrophy and disability in multiple sclerosis over four years: application of a reproducible automated technique in monitoring disease progression in a cohort of the interferon beta-1a (Rebif) treatment trial. J Neurol Neurosurg Psychiatry 74:1090-1094

13. Rocca MA, Valsasina P, Meani A et al (2019) Clinically relevant cranio-caudal patterns of cervical cord atrophy evolution in MS. Neurology 93:e1852-e1866

14. Bonacchi R, Pagani E, Meani A et al (2020) Clinical relevance of multiparametric MRI assessment of cervical cord damage in multiple sclerosis. Radiology 296:605-615

15. Lukas C, Knol DL, Sombekke MH et al (2015) Cervical spinal cord volume loss is related to clinical disability progression in multiple sclerosis. J Neurol Neurosurg Psychiatry 86:410-418

16. Lukas C, Sombekke MH, Bellenberg B et al (2013) Relevance of spinal cord abnormalities to clinical disability in multiple sclerosis: MR imaging findings in a large cohort of patients. Radiology 269:542-552

17. Moccia M, Ruggieri S, Ianniello A, Toosy A, Pozzilli C, Ciccarelli O (2019) Advances in spinal cord imaging in multiple sclerosis. Ther Adv Neurol Disord. https://doi.org/10.1177/ 1756286419840593.eCollection2019

18. Kappos L, Wolinsky JS, Giovannoni G et al (2020) Contribution of relapse-independent progression vs relapse-associated worsening to overall confirmed disability accumulation in typical relapsing multiple sclerosis in a pooled analysis of 2 randomized clinical trials. JAMA Neurol 77:1132-1140

19. Lorscheider J, Buzzard K, Jokubaitis V et al (2016) Defining secondary progressive multiple sclerosis. Brain 139:2395-2405

20. Weiler F, Hallmann MF, Schwier M et al (2017) Fully automated detection, segmentation and quantification of mean crosssectional area of the spinal cord. Mult Scler 23(S3):149-150

21. Gregori J, Cornelissen C, Hoffmann S et al (2018) Feasibility of fully automated atrophy measurement of the upper cervical spinal cord for group analyses and patient- individual diagnosis support in MS. Mult Scler 24(S2):623-624

22. Eden D, Gros C, Badji A et al (2019) Spatial distribution of multiple sclerosis lesions in the cervical spinal cord. Brain 142:633-646
23. De Leener B, Fonov VS, Collins DL, Callot V, Stikov N, CohenAdad J (2018) PAM50: unbiased multimodal template of the brainstem and spinal cord aligned with the ICBM152 space. Neuroimage 165:170-179

24. De Leener B, Levy S, Dupont SM et al (2017) SCT: Spinal Cord Toolbox, an open-source software for processing spinal cord MRI data. Neuroimage 145:24-43

25. Smith SM, De Stefano N, Jenkinson M, Matthews PM (2001) Normalized accurate measurement of longitudinal brain change. J Comput Assist Tomogr 25:466-475

26. Smith SM, Zhang Y, Jenkinson M et al (2002) Accurate, robust, and automated longitudinal and cross-sectional brain change analysis. Neuroimage 17:479-489

27. Smith SM, Jenkinson M, Woolrich MW et al (2004) Advances in functional and structural MR image analysis and implementation as FSL. Neuroimage 23(Suppl 1):S208-219

28. Clarke MA, Pareto D, Pessini-Ferreira L et al (2020) Value of 3T susceptibility-weighted imaging in the diagnosis of multiple sclerosis. AJNR Am J Neuroradiol 41:1001-1008

29. Frischer JM, Weigand SD, Guo Y et al (2015) Clinical and pathological insights into the dynamic nature of the white matter multiple sclerosis plaque. Ann Neurol 78:710-721

30. Luchetti S, Fransen NL, van Eden CG, Ramaglia V, Mason M, Huitinga I (2018) Progressive multiple sclerosis patients show substantial lesion activity that correlates with clinical disease severity and sex: a retrospective autopsy cohort analysis. Acta Neuropathol 135:511-528

31. Maggi P, Sati P, Nair G et al (2020) Paramagnetic rim lesions are specific to multiple sclerosis: an international multicenter 3T MRI study. Ann Neurol 88:1034-1042

32. Kutzelnigg A, Lucchinetti CF, Stadelmann C et al (2005) Cortical demyelination and diffuse white matter injury in multiple sclerosis. Brain 128:2705-2712

33. Preziosa P, Pagani E, Moiola L, Rodegher M, Filippi M, Rocca MA (2020) Occurrence and microstructural features of slowly expanding lesions on fingolimod or natalizumab treatment in multiple sclerosis. Mult Scler. https://doi.org/10.1177/1352458520969105

34. van Waesberghe JH, Kamphorst W, De Groot CJ et al (1999) Axonal loss in multiple sclerosis lesions: magnetic resonance imaging insights into substrates of disability. Ann Neurol 46:747-754

35. Kearney H, Altmann DR, Samson RS et al (2015) Cervical cord lesion load is associated with disability independently from atrophy in MS. Neurology 84:367-373

36. Enzinger C, Barkhof F, Ciccarelli O et al (2015) Nonconventional MRI and microstructural cerebral changes in multiple sclerosis. Nat Rev Neurol 11:676-686

Publisher's note Springer Nature remains neutral with regard to jurisdictional claims in published maps and institutional affiliations. 